\title{
Vehicle Tracking and Locking System Using GPS and GSM
}

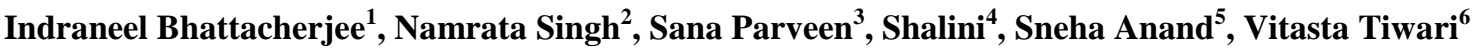 \\ Student, $4^{\text {th }}$ Year, Electrical and Electronics Engineering, IMS Engineering College, Ghaziabad, India ${ }^{1,2,3,4,5,6}$
}

\begin{abstract}
In this paper, we presented a vehicle tracking system using GPS modem and GSM module and locking it down using remote mobile accessing. The system is designed to provide security to vehicle owners from theft and unauthorized access. The system performs multiple operations which include:-When an unauthorized person tries to gain access of your vehicle, the information is analyzed using sensors and transmitted to user through GSM. The coordinates of the location of vehicle are sent via GPS. The controlling of vehicle is done through microcontroller. The doors of the vehicles and accelerator pedal are locked down when reading instructions from the user. The speed of the motor is gradually reduced and comes to a halt. By using the control concepts listed in the paper number of control features can be implemented in the same fashion.
\end{abstract}

Index Terms: GPS, GSM, sensors, Speed control, Tracking, Vehicle locking, Vehicle control.

\section{INTRODUCTION}

The vehicle tracking system is an electronic device that assembly of components will be discussed in tracks the vehicle's location. Most of the tracking system implementation section, (v) conclusion, advantages and uses GPS module to locate vehicles position [1]. Many future scope.

systems also combine communication components such as satellite transmitters to communicate the vehicle's location to the remote user. Google maps are used to view vehicle's location. The design of the tracking system has been divided into 3 parts: basic design, intermediate design and advanced design.

The basic design of the vehicle tracking system consists of a GSM module, GPS module, a microcontroller, a relay circuit and a LCD. In this paper we have included the sensors to detect the unauthorized intervention. When any unauthorized user (i.e. a person other than the owner) tries to get access of vehicle, the system sends an SMS to owner. The user commands the system to lock down the vehicle remotely. The gates and accelerator pedal are locked down using instructions from the microcontroller. The speed of the motor is gradually reduced. Meanwhile the coordinates of the location (i.e. latitude and longitude) are sent to users mobile. These coordinates can be used to view the location of vehicle on Google Maps. This system designed for users in land construction and transport business, provides real-time information such as location, speed and expected arrival time of the user is moving vehicles in a concise and easy-to-read format. This system may also useful for communication process among the two points.

Currently GPS vehicle tracking ensures their safety as travelling [2]. This vehicle tracking system found in clients vehicles as a theft prevention and rescue device. Vehicle owner or Police follow the signal emitted by the tracking system to locate a robbed vehicle. The paper is divided into following sections: (i) researches carried out so far, (ii) components being used in the system, (iii) hardware and software designing process, (iv) the

\section{SURVEY OF RELATED RESEARCH}

Number of papers has been published on the development of vehicle tracking system using GPS and GSM modem $[3],[4],[5],[6],[7],[8],[9],[10],[11],[12],[13]$ and [14]. In [15] differential GPS algorithm that is capable of providing real time near PPP service is presented. In [16] error sources in GPS measurement are calculated. In [17] development of real-time visual tracking system for vehicle safety applications is discussed and the concept of focus of expansion (FOE) is introduced. A low cost real time tracking system that provides accurate localizations of the tracked vehicle is presented in [18].Vehicle tracking coupled with vehicle registration number recognition is introduced in [19]. Following huge demand of accurate vehicle tracking systems, researchers proposed number of novel methods [20], [21], [22] to improve the accuracy of tracking system.

In [23], the hardware and software of the GPS and GSM network were developed. The proposed GPS/GSM based System has the two parts, first is a mobile unit and another is controlling station. The system processes, interfaces, connections, data transmission and reception of data among the mobile unit and control stations are working successfully. These results are compatible with GPS technologies.

In [24], a vehicle tracking system is an electronic device, installed in a vehicle to enable the owner or a third party to track the vehicle's place. This paper proposed to design a vehicle tracking system that works using GPS and GSM technology. This system built based on embedded system, used for tracking and positioning of any vehicle by using 
Global Positioning System (GPS) and Global system for mobile communication (GSM). This design will continuously watch a moving Vehicle and report the status of the Vehicle on demand.

In [25], Face Detection System used to detect the face of the driver, and compare with the predefined face. The car owner is sleeping during the night time and someone theft the car. Then Face Detection System obtains images by one tiny web camera, which is hidden easily in somewhere in the car. Face Detection System compared the obtained images with the stored images. If the images don't match, then the information sends to the owner through MMS. The owners get the images of the thief in mobile phone and trace the place through GPS. The place of the car and its speed displayed to the owner through SMS. The owner can recognize the thief images as well as the place of the car and can easily find out the hijackers image. This system applied in our day-to-day life.

In [26], this system provided vehicle cabin safety, security based on embedded system by modifying the existing modules. This method monitors the level of the toxic gases such as CO, LPG and alcohol within the vehicle provided alert information as alarm during the dangerous situations. The SMS sends to the authorized person through the GSM. In this method, the IR Sensor used to detect the static obstacle in front of the vehicle and the vehicle stopped if any obstacle detected. This is avoiding accidents due to collision of vehicles with any static obstacles.

\section{COMPONENTS USED}

\section{A. The Microcontroller}

There are two ways to control an electronic circuit either using: Microprocessor or MCU. The Microprocessors are usually referred to as general purpose microprocessors because they do not contain RAM, ROM and I/O ports. So, system designers have to add an external RAM, ROM and $\mathrm{I} / \mathrm{O}$ ports to make a system functional. Addition of these components will make the system bulkier and much more expensive. The advantage of using microprocessor is that the designer can decide the amount of RAM, ROM and $\mathrm{I} / \mathrm{O}$ ports needed to accomplish a task.

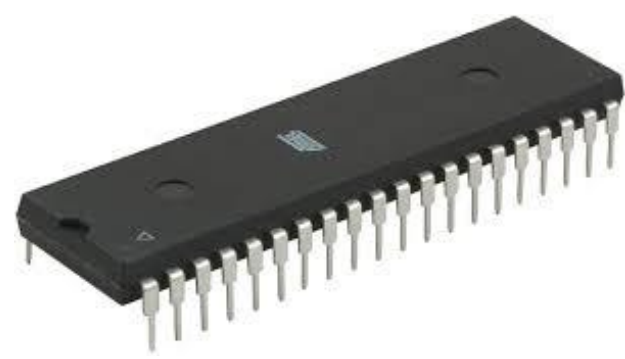

Fig 1: Microcontroller Chip

However, MCUs have a CPU in addition to the fixed amount of RAM, ROM and I/O ports, which are embedded on a chip with support functions such as a crystal oscillator, timers and serial or analog input output (I/O) [27]. The MCUs are designed for embedded applications and can be used in remote controls, power tools, toys and other appliances. Invention of MCUs has reduced the size and cost of designs. MCUs are suitable where cost and space are critical. There are four types of MCUs ( 8 bit): 8051 family, PIC, Zilog and Free scale. The MCU families are not compatible with each other, which means, if we write a code for $8051 \mathrm{MCU}$ it will not work on PIC MCU. This is mainly due to different instructions and registers set in each MCU.

To choose among these MCUs, there are specific criteria set for designers: MCU should meet the task at hand efficiently and cost effectively [28]. Software development tools such as compilers, assemblers and debuggers should be available in the market. Wide availability and reliable sources of the MCU used. Designer should also consider the speed, Packaging, power consumption, the amount of RAM, ROM on chip and cost per unit [29].

\section{B. GSM Modem}

A GSM module is a second generation digital mobile cellular technology, which covers a fairly broad geographical area. This offers customized travel, financial, reference and commercial information to the users [30]. It can operate in $400 \mathrm{MHz}, 900 \mathrm{MHz}$ and $1800 \mathrm{MHz}$ frequency bands. The GSM modem can accept a SIM card just like a mobile phone and operate on a subscription to a network of mobile data transfer. The GSM Modem supports three types of services namely bearer or data services, supplementary services, and telecommunication services. A typical GSM picture is given below:

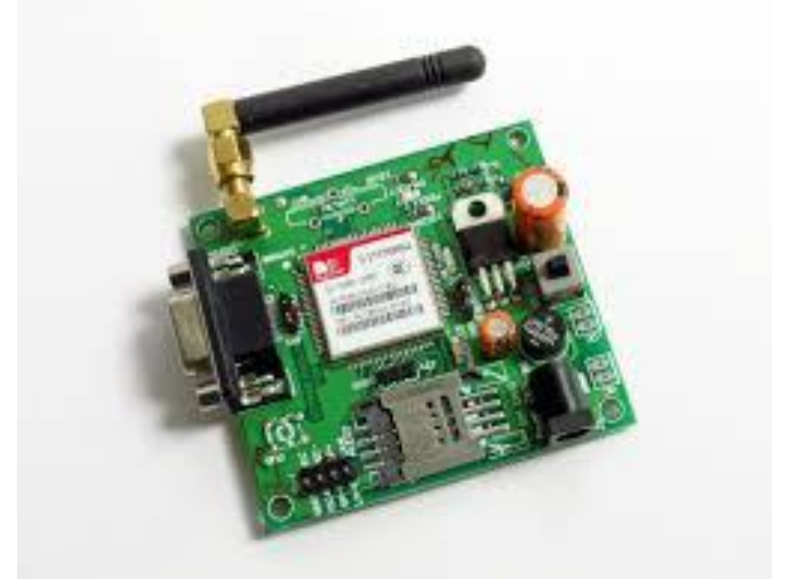

Fig 2: GSM module

\section{GPS Module}

The first GPS (navigation system) was designed by Honda in 1983. Pioneer claims to be the first with a GPS-based auto navigation system, in 1990. Magellan claims to have created the first GPS-based vehicle navigation system in the U.S in 1995. Each GPS (satellite) transmits data that indicates the current time and its location. It transmits signals to a GPS receiver. This receiver requires an unobstructed view of the sky, so they can only be used effectively outdoors. The step by step operation of the GPS can be seen in figure 3 . 
Vol. 4, Issue 2, February 2017

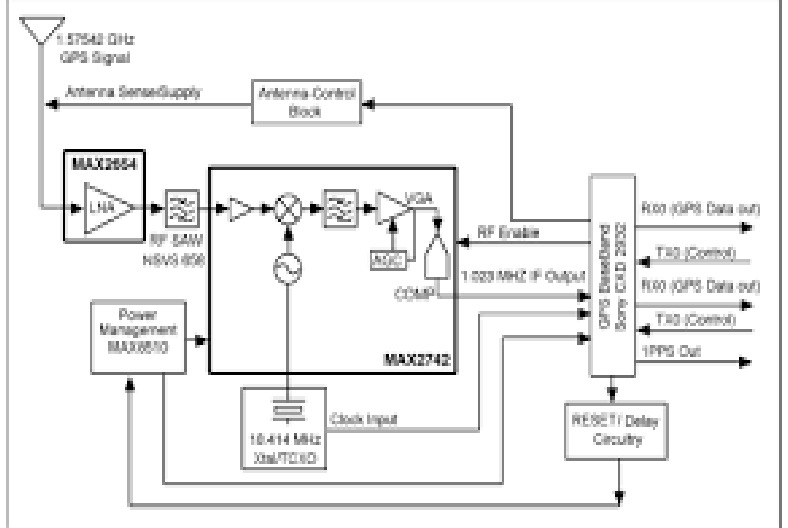

Fig 3: Operation of GPS

\section{Sensors}

Different types of sensors can be employed to detect the unauthorized presence. It could be an infrared sensor, vibration sensor etc.

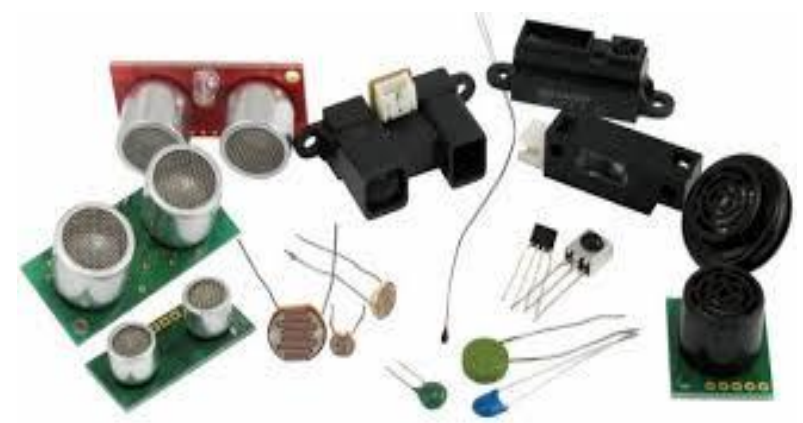

Fig 4: Different types of sensors

\section{SYSTEM DESIGNING}

\section{A. Basic Designing}

Basic design will allow users to send a SMS from a mobile phone to the tracking system and get the response back via SMS. The user will input the longitude and latitude received in a SMS to the designed web page to view the vehicle's location on Google maps. The basic design of the vehicle tracking system consists of a GSM module, a GPS module, a MCU (ATMEL), a relay circuit and a LCD. The figure shown below represents the block diagram of the components used in order to build the vehicle tracking system. First of all, the system will be initialized by sending a SMS to the GSM Modem. The GSM modem will read the SMS and transmit to the MCU unit. The MCU unit will process the request by first checking the mobile number against the stored numbers in its library. If a mobile number matches to the MCU library number, the user request will be further processed by first comparing the request code with the stored codes and then taking the appropriate actions.

\section{B. Advanced Designing}

The advance design will allow the users to track the vehicle's location by just plugging a small device (like USB) to the computer. The system will automatically send the request to the tracking system to get the location and display the received data on Google maps. The advance design of the vehicle tracking system consists of a MAX232, a MCU (ATMEL), a GSM Modem and an IC. The serial port (MAX232 and the RS232) is used to connect the advance design to the computer. The figure shown below represents the block diagram of the components used in order to build the vehicle tracking system. The AT commands are used to make the advance design work. Once the device is plugged to the computer, it will automatically run the system. The computer (via device) will send a SMS to the device and the device will respond in its usual fashion (Respond back via SMS). In the basic and advance designs, the user has to request the location of the device but the advance design offers the plug \& play feature.

\section{Proposed Designing}

In this proposed work, a novel method of vehicle tracking and locking system used to track the theft vehicle by using GPS and GSM technology. This system puts into sleeping mode while the vehicle handled by the owner or authorized person otherwise goes to active mode, the mode of operation changed by in person or remotely. If any interruption occurred in any side of the door, then the IR sensor senses the signals and SMS sends to the microcontroller. The controller issues the message about the place of the vehicle to the car owner or authorized person. When send SMS to the controller, issues the control signals to the engine motor. Engine motor speeds are gradually decreases and come to the off place. After that all the doors locked. To open the door or restart the engine, authorized person needs to enter the passwords. In this method, tracking of vehicle place easy and doors locked automatically, thereby thief cannot get away from the car. The circuit diagram of the vehicle tracking and locking embedded system using GPS and GSM technology is shown in Fig.3.The compact circuitry is built around Atmel AT89C52 microcontroller.

The AT89C52 is a low power; high performance CMOS 8-bit microcomputer with $8 \mathrm{kB}$ of Flash programmable and erasable read only memory (PEROM). It has 256 bytes of RAM, 32 input/output (I/O) lines, three 16-bit timers/ counters, a six-vector two-level interrupt architecture a full-duplex serial port, an on-chip oscillator and clock circuit. The system clock also plays a significant role in operation of the microcontroller. An $11.0592 \mathrm{MHz}$ quartz crystal connected to pins 18 and 19 provides basic clock to the microcontroller. Power-on reset is provided by the combination of electrolytic capacitor $\mathrm{C} 3$ and resistor R1. Port pins P2.0 through P2.7 of the microcontroller are connected to data port pins D0 through D7 of the LCD, respectively. Port pins P0.5, P0.6 and P0.7 of the microcontroller are connected to Register-select (RS), Read / write (RW) and enable (E) pins of the LCD, respectively. All the data is sent to the LCD in ASCII format for display. Only the commands are sent in hex form. Register-select (RS) signal is used to distinguish between data $(\mathrm{RS}=1)$ and command $(\mathrm{RS}=0)$. Preset $\mathrm{RV} 1$ is used to control the contrast of the LCD. Resistor $10 \mathrm{k}$ 
Vol. 4, Issue 2, February 2017

limits the current through the backlight of the LCD. Port detecting the unauthorized person. Port1.7 is used to pins P3.0 (RXD) and P3.1 (TXD) of the microcontroller connect the alarm through transistor BC547, Pin number are used to interface with the RFID reader through P3.3 is used to connect the engine motor. If unauthorized Max232 and GSM Modem are used to interface through person enter into the car, the IR sensor sense the signals if Max232. Port pins from P1.0 to P2.7 of the any interruption occurs in any side of the door and send to microcontroller are connected to keyboard. The GPS and the microcontroller, then the controller issue the message GSM are used to connect through RXD and TXD pins of about the location of the vehicle to car owner or the microcontroller for further processing. The port pins of authorized person.

P0.0 to P0.3 are used to connect the 4 IR sensors for

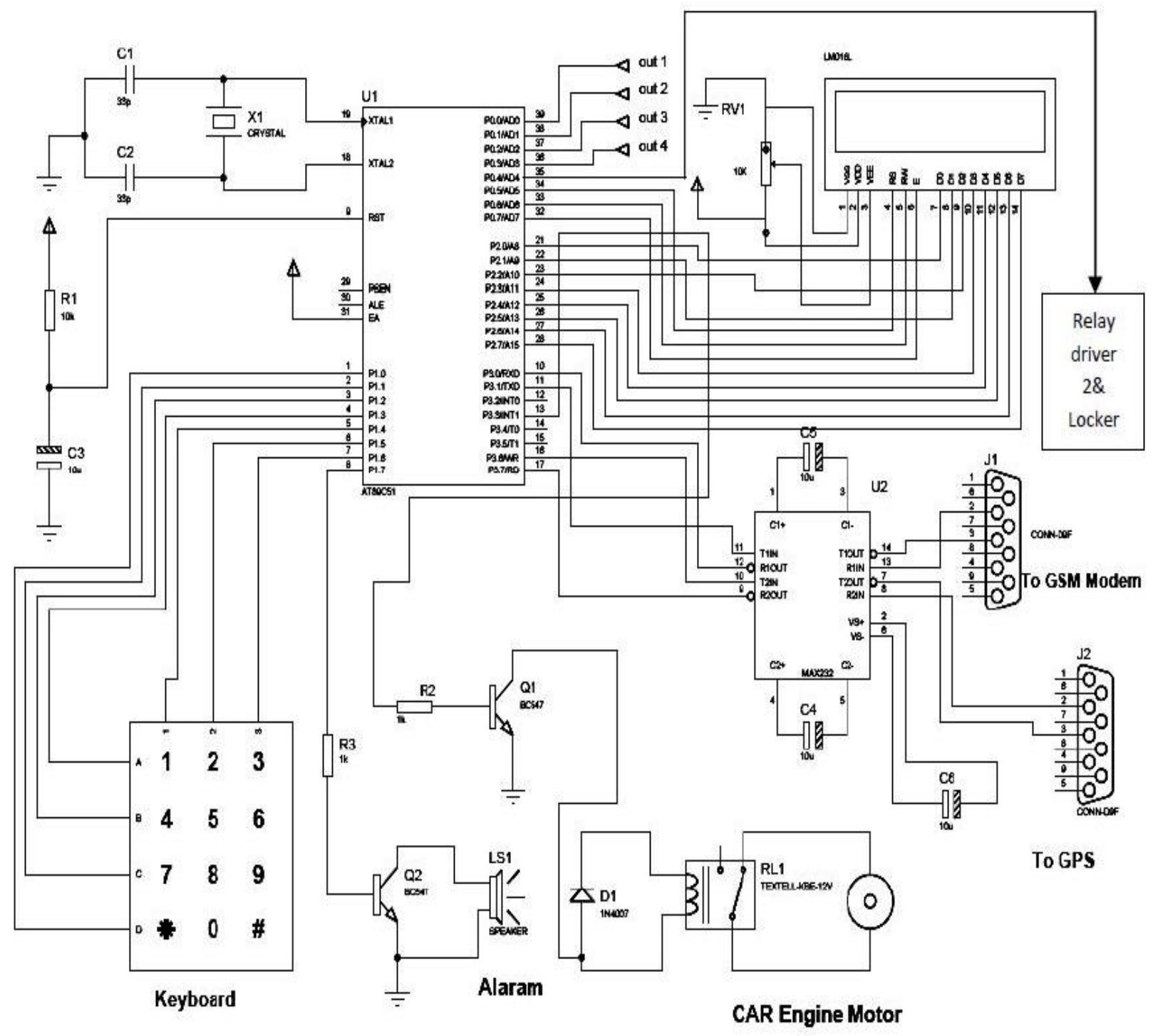

Fig 5: Circuit Diagram of Vehicle Locking and Tracking System

When send the SMS to controller, issues the control signals to the engine motor. Engine motor speed is gradually decreases and comes to the off position. After that all the doors are locked. Pin P0.4 is used to connect the door locker through relay. To open the door or to restart the engine authorized person needs to enter the beh passwords. In this method, tracking of vehicle location easy and also doors are locked automatically thereby thief cannot get away from the car. Pin P0.4, transistor Q3 drives into saturation, and relay RL2 energizes to close or open the door lock.

\section{IMPLEMENTATION PROCESS}

In the implementation section, we will discuss the steps that were followed to build the vehicle tracking system. Furthermore, this chapter will also focus the reasons behind choosing specific components used in the tracking system.

Following steps were taken to build the system: Selecting the right component, choosing the programming language and appropriate software to simulate the design. 
Vol. 4, Issue 2, February 2017

\section{A. Selecting the right components}

An extensive research has been carried out to find the right components to build the vehicle tracking system. Some of the components used are listed below with a brief description.

The MCU Unit: Each MCU has a unique functionality, which can make them more appropriate for use over the rest. For the basic design and advance design, AT89S52 MCU from 8051 family was used. The AT89S52 is a lowpower, high performance CMOS 8-bit MCU with 8K bytes of in system programmable flash memory.

Liquid Crystal Diode (LCD): Dot Matrix LCD 16*2 (16 char \& 2 rows) is used. It can display the location of a vehicle in terms of coordinates and the SMS sent or received by the GSM modem. The two rows of the LCD are used to show the north and east coordinate. The Pin 2 is connected to VCC and pin1 with Ground. Pin no 3 is connected with resistor value of $10 \mathrm{~K}$ Variable Resistor that is used for the contrast colour of the LCD. Pin no 4 (RS), 5(R/W), 6(EN) are attached with P2.5, P2.6, P2.7 of the MCU respectively. Rests of the 8 pins are attached to port 0 of the MCU.

Oscillator: The speed of the MCU depends on the frequency of the oscillator. There are oscillators of different frequencies available. An oscillator of 11.0592 $\mathrm{MHz}$ frequency is chosen. Some of the components (i.e. oscillators) used in this project were available in the local lab and simply chosen because of their urgent availability. The MAX232 is used to connect the MCU with RS232, which fits into the serial port of the computer.

The GSM: A GSM modem used in this project is SIMCOM SIM 300DZ. The GSM modem (SIM 300DZ) is tri-band GPRS/GSM device. This can operate over 900, 1800 and $1900 \mathrm{MHz}$ bands. The SIM 300DZ provides RF antenna interface. With the charge circuit integrated inside the GSM module (SIM 300DZ), it is very suitable for the battery power application. With a dimension of $33 \times 33 \times$ $3 \mathrm{~mm}$, it can fit in almost all the wireless applications. The SIM card is inserted for the purpose of the user identification in the GSM module. The GPS One of the most important tasks in the project is designing and implementation of the GPS receiver circuit.

\section{B. Choosing the right programming language}

Although, there are lot of programming languages that could be used to program the MCU, the most common ones are $\mathrm{C}$ programming language and Assembly language. The programs written in the assembly language can execute faster, while programs written in the $\mathrm{C}$ programming are easier to develop and maintain. I have used the assembly language in 2nd year and 3rd year of my engineering projects. So, I have better command and knowledge of the assembly language. Therefore; assembly language was chosen to build the vehicle tracking system. Web Page to track and control vehicle HTML language is used to develop the webpage needed to display the location of a vehicle. The Google maps are embedded in my webpage so, that the user can input the longitude and latitude to view the location. .

\section{Appropriate software to simulate my design}

Although there are varieties of software packages, which can be used to simulate the circuit; the most commonly used are the circuit wizard and the PCB wizard. In order to test the circuit, Proteus design suite (software) is used. It is very powerful tool for the electronic circuit simulation, the schematic capture and the printed circuit board (PCB) design. By combining ISIS schematic capture and ARES PCB layout the Proteus design suite (software) provides an integrated and GPS based vehicle Tracking and Vehicle Control System.

\section{CONCLUSION}

In this paper, we have proposed a novel method of vehicle tracking and locking systems used to track the theft vehicle by using GPS and GSM technology. This system puts into the sleeping mode vehicle handled by the owner or authorized persons; otherwise goes to active mode. The mode of operations changed by persons or remotely. When the theft identified, the responsible people send SMS to the micro controller, then issue the control signals to stop the engine motor. After that all the doors locked. To open the doors or to restart the engine authorized person needs to enter the passwords. In this method, easily track the vehicle place and door is locked.

\section{ACKNOWLEDGEMENT}

The authors would like to sincerely thank Dr. Rishi Asthana sir, HOD, Electrical and Electronics department and our mentor Mayank Agarwal sir, Assistant Professor, Electrical and Electronics department, IMS Engineering College for their valuable support and help in this research.

\section{REFERENCES}

[1] EI-Rabbany, Introduction to GPS: The Global Positioning System, Norwood, MA: Artech House, 2006.

[2] M. Brain, "How Microcontroller Works," HowStuffWorks, a division of InfoSpace LLC, [Online]. Available: http://www.howstuffworks.com/microprocessor.[Accessed $10 \quad 12$ 2014].

[3] H. D. Pham, M. Drieberg and C. C. Nguyen, "Development of vehicle tracking system using GPS and GSM modem," in IEEE Conference on Open Systems (ICOS), Kuching, 2013.

4] M. Ahmad Fuad and M. Drieberg, "Remote vehicle tracking system using GSM Modem and Google map," in IEEE Conference on Sustainable Utilization and Development in Engineering and Technology (CSUDET), Selangor, 2013.

5] M. Parvez, K. Ahmed, Q. Mahfuz and M. Rahman, "A theoretical model of GSM network based vehicle tracking system," in International Conference on Electrical and Computer Engineering (ICECE), Dhaka, 2010.

[6] R.Ramani, S.Valarmathy, D. N.SuthanthiraVanitha, S.Selvaraju and M.Thiruppathi.R.Thangam, "Vehicle Tracking and Locking System Based on GSM and GPS," I.J. Intelligent Systems and Applications, vol. 09, pp. 89-93, August 2013

[7] P. P. Wankhade and P. S. Dahad, "Real Time Vehicle Locking and Tracking System using GSM and GPS Technology-An Anti-theft System," International Journal of Technology And Engineering System(IJTES), vol. 2, no. 3, 2011.

[8] P. Verma and J. Bhatia, "Design and Development of GPSGSM based Tracking System with Googlemap based Monitoring," 
Vol. 4, Issue 2, February 2017

International Journal of Computer Science, Engineering and Applications (IJCSEA), vol. 3, no. 2, June 2013.

[9] T. Le-Tien and V. Phung-The, "Routing and Tracking System for Mobile Vehicles in Large Area," Fifth IEEE International Symposium on Electronic Design, Test and Application, pp. 297300, January 2010.

[10] P. Fleischer, A. Nelson, R. Sowah and A. Bremang, "Design and development of GPS/GSM based vehicle tracking and alert system for commercial inter-city buses," IEEE 4th International Conference on Adaptive Science \& Technology (ICAST), October 2012.

[11] M. N. Ramadan, M. A. Al-Khedher and S. A. Al-Kheder, "Intelligent Anti-Theft and Tracking System for Automobile," International Journal of Machine Learning and Computing, vol. 2, no. 1, February 2012.

[12] D. A. Brown, "A Low Cost Vehicle Location and Tracking System," NAVSYS Corporation, pp. 516-523, 1992.

[13] M. A. Elahi, Y. A. Malkani and M. Fraz, "Design and implementation of real time vehicle tracking system," 2nd International Conference on Computer, Control and Communication, pp. 1-5, 2009. [14] P. A. Okatan and A. Salih, "Micro-Controller based Vehicle Tracking System," IEEE, pp. 605609, 2003.

[14] H.-W. Chen, Y.-T. Chiang, F.-R. Chang and H.-S. Wang, "Toward real-time precise point positioning: Differential GPS based on IGS ultra rapid product," in Proceedings of IEEE in SICE Annual Conference, 2010

[15] P. Misra and P. Enge, "Global Positioning System: Signals, Measurements, and Performance," in Massachusetts, GangaJamuna Press, 2001.

[16] K.-T. Song and C.-C. Yang, "Front Vehicle Tracking Using Scene Analysis," in Proceedings of the IEEE International Conference on Mechatronics \& Automation, 2005.

[17] W. El-Medany, A. Al-Omary, R. Al-Hakim, S. Al-Irhayim and M. Nusaif, "A Cost Effective Real-Time Tracking System Prototype Using Integrated GPS/GPRS Module," in 6th International Conference on In Wireless and Mobile Communications (ICWMC), 2010

[18] H. Lee, D. Kim, D. Kim and S. Y. Bang, "Real-time automatic vehicle management system using vehicle tracking and car plate number identification," in International Conference on Multimedia and Expo. ICME '03, 2003.

[19] Dewandaru, A. M. Said, A. N. Matori and U. T. Petronas, "A Novel Map-matching Algorithm to Improve Vehicle Tracking System Accuracy," 2007.

[20] G. Challita, S. Mousset, F. Nashashibi and A. Bensrhair, "An application of $\mathrm{V} 2 \mathrm{~V}$ communications : Cooperation of vehicles for a better car tracking using GPS and vision systems," in IEEE Vehicular Networking Conference (VNC), 2009.

[21] "The Microprocessors," Connect in, 08 February 2005.

[22] Asaad M. J. Al-Hindawi, Ibraheem Talib, "Experimentally Evaluation of GPS/GSM Based System Design", Journal of Electronic Systems Volume 2 Number 2 June 2012

[23] Kunal Maurya , Mandeep Singh, Neelu Jain, "Real Time Vehicle Tracking System using GSM and GPS Technology- An Anti-theft Tracking System," International Journal of Electronics and Computer Science Engineering.

[24] Vikram Kulkarni \& Viswaprakash Babu, "embedded smart car security system on face detection', special issue of IJCCT,

[25] V.Ramya, B. Palaniappan, K. Karthick, "Embedded Controller for Vehicle In-Front Obstacle Detection and Cabin Safety Alert System", International Journal of Computer Science \& Information Technology (IJCSIT) Vol 4, No 2, April 2012.

[26] "Introduction, MCU," Astronomy pocket Pc Project, 2009. [Online].Available:http://astrosurf.com/astroenit/projets/ast ronomy_pocket_pc_en.

[27] "Introduction to Microcontrollers," Society of Robots, September 2005. [Online]. Available: http://www.societyofrobots.com/micro controller_tutorial.shtml. [Accessed 1212 2014].

[28] "The 8051 microcontroller architecture," Electronic and Electricity, February 2010. [Online]. Available: http://www.zonetronik.com/en/ 2010/01/the-8051- microcontroller-architecture. [Accessed 1310 2010].

[29] M. A. Mazidi, J. Mazidi and R. McKinlay, The 8051 Microcontroller and Embedded Systems (2nd edition), Prentice Hall, 2006. 